\title{
M. FOUCAULT: LA PREGUNTA POR EL PODER
}

\author{
Patxi Lanceros
}

Para una lectura apresurada y ciertamente superficial, el poder sería el concepto central de la filosofía foucaultiana, el objeto - finalmente hallado- gracias al cual se recuperan unos primeros pasos titubeantes y alguna que otra reflexión despistada. Los primeros serían los pasos dados en pos de una tematización de la locura y la enfermedad, problemas ambos «políticamente sin importancia y epistemológicamente sin nobleza» ${ }^{1}$. Las segundas aludirían al esfuerzo invertido en la polémica narración de una «historia de las ciencias» (Las palabras y las cosas) que, a la vista del trabajo posterior, no sería sino un paréntesis, una especie de capricho incrustado en la «verdadera» trayectoria foucaultiana; la que desde el principio acosa al poder, hostiga sus múltiples formas de aparición, desvela sus secretos, muestra sus maniobras y su injustificable insidia.

Es cierto que alguna frase de Foucault puede justificar esta percepción. Un ejemplo: respondiendo a una pregunta de M. Fontana en la que éste señala a Foucault que él ha sido el primero en «plantear al discurso la cuestión del poder», el filósofo francés afirma: «No pienso haber sido el primero en plantear esa cuestión. Al contrario, estoy sorprendido de la dificultad que tuve para formularla. Cuando lo pienso de nuevo, ahora, me pregunto, ¿de qué he podido hablar, por ejemplo, en la Historia de la locura, o en El Nacimiento de la clínica, si no era del poder?»².

La apreciación que, bien entendida, puede sugerir una interesante perspectiva de lectura de las primeras obras de Foucault, ha servido sin embargo para abonar la imagen de un pensador monolíticamente centra-

1 Foucault, M.: «Verdad y Poder». Entrevista con M. Fontana. En Microfísica del Poder. Ed. La Piqueta, Madrid, 1980 (MP), p. 176.

2 Ibid., p. 180. 
do en el estudio del poder; de un pensamiento unívocamente referido al poder e incluso obsesionado por él ${ }^{3}$.

Incluso el nuevo ordenamiento de la obra foucaultiana que hoy es común, ha servido — salvo excepciones — ${ }^{4}$ para dar mayor credibilidad a la imagen de un Foucault casi ininterrumpidamente concernido por el estudio del poder ${ }^{5}$ : una vez desahuciado el prestigio metodológico de la arqueología, obras que antaño contaron entre las más presentables conquistas del método arqueológico y su aplicación al saber, pasan ahora a engrosar la nómina de estudios sobre el poder. Según esto, la Historia de la locura y El Nacimiento de la clínica ya no hablarían del saber psicológico-psiquiátrico y de su lenta elaboración, tampoco de la medicina en cuanto ciencia. El objeto sería institucional: el manicomio, el hospital. El objeto sería la institución que, a la luz de los estudios posteriores, vendría a incluirse en el «archipiélago carcelario»: una especie de prólogo - por lo tanto- al texto al que habitualmente se dirige la atención cuando de poder y Foucault se habla, Vigilar y Castigar: el nacimiento de la prisión.

La perspectiva que vamos a sostener en las siguientes páginas no sólo rechaza la anteriormente esbozada, sino que pretende oponerse abiertamente a ella. Resumimos en breves trazos los elementos fundamentales que posteriormente intentaremos articular:

- Constatar la presencia en la obra de Foucault de múltiples referencias al poder y a los poderes, al ejercicio del poder, a los efectos, tipos y formas de poder, etc..., no basta para concluir que el poder es el tema general, el objeto central, de su trabajo. Hay que preguntar aún más. Hay que interrogar, como el propio Foucault hace, ¿Por qué estudiar el poder ${ }^{6}$ A esta pregunta el mismo autor responde con una escueta afirmación: la cuestión del sujeto, y confirma, aun con evidente laconismo: «No es el poder lo que constituye el tema general de mis investigaciones sino el sujeto» ${ }^{7}$.

- Cuando Foucault estudia el poder, apenas se pregunta ¿qué es? sino por el contrario «¿cómo se ejerce? Parece no interesarle la

3 Berman, M.: Todo lo sólido se desvanece en el aire. Ed. Siglo XXI, Madrid, 1988, p. 24.

${ }^{4}$ Entre ellas cabe mencionar a M. MoreY, atento a la totalidad de la trayectoria foucaultiana, en el prólogo a la obra de Foucault Tecnologías del yo.

5 Entre otros Dews, P.: Power and Subjectivity in Foucault, New Left Review 144, march-april 1984, pp. 72-95; ALLEN, B.: «Government in Foucault», Canadian journal of philosophy. Vol. 21, Number 4. December 1991, pp. 421-440; Berman, M.: Op. cit.; BREUER, S.: Die Gesellschaft des Verschwindens, Junius Verlag, Hamburg, 1993.

${ }^{6}$ Foucault, M.: «The Subject and Power», in Hubert L. DreYfus and PaUl Rabinow: Michel Foucault, Beyond Structuralism and Hermeneutics, University of Chicago Press, 1982, pp. 208 ss.

7 Ibid., p. 209. 
esencia del poder, acaso porque no haya tal esencia. Parece interesarle más el ejercicio que el Poder, acaso porque no haya tal $\mathrm{Po}$ der. «En términos bruscos diría que referir el análisis del poder al cómo es introducir la sospecha de que el Poder no existe; es preguntarse en todo caso cuáles son los contenidos asignables que se avistan cuando se usa ese término majestuoso, globalizante y substantificador; es suponer que se deja escapar un conjunto de realidades tremendamente complejas cuando uno se somete indefinidamente a la doble interrogación ¿Qué es el Poder? ¿De dónde viene el Poder?» ${ }^{8}$.

-El tratamiento que Foucault hace de los «tipos de poder», de las «formas históricas de ejercer el poder», no pertenece a la pléyade de opciones teóricas que compiten en el ámbito de la Teoría política. Para todas ellas -independientemente de sus presupuestos e inclinaciones- el Poder existe al margen de sus condiciones históricas de ejercicio: se trataría de una especie de invariante conceptualmente atrapable que sobrevuela la historia y se metamorfosea en lo que respecta a las formas externas: instrumentos que usa, instituciones en las que se localiza, instancias de legitimación a las que invoca, etc... Para Foucault — nominalista, pluralista - no hay necesidad ni posibilidad de reducir la multiplicidad de poderes a la presunta unidad del Poder. No cabe el argumento de corte trinitario que sometería la variedad de poderes distintos al único Poder verdadero.

-Finalmente: Foucault sugiere un estudio concertado de las distintas prácticas de poder (dominio, gobierno, disciplina, normalización) y los distintos juegos de verdad (saber). Del conjunto saber-poder surgen las tecnologías (que pueden tener carácter institucional pero no es necesario que lo adquieran) en las que se constituye el sujeto visto desde la óptica de las relaciones entre los hombres (individuos y/o grupos). De este enunciado se siguen dos importantes conclusiones:

a) No hay subsunción del poder por el saber ni viceversa: ambos son autónomos y co-relativos.

b) El poder no es algo que le llega al sujeto desde el exterior: algo que se tiene, que se conquista o que se pretende. El poder es el universo de relaciones en el que se está. El conjunto de relaciones que constituyen sujeto (en un doble sentido que esclareceremos). 
Estos cuatro puntos pueden servir como introducción a la lectura de algunos textos de Foucault que ahora iniciamos. A lo largo de ella no nos vamos a detener en determinados momentos de los cuales la crítica ha hecho emblema. Tampoco nos proponemos mostrar los esquemas teóricos de los cuales Foucault sería más o menos deudor: el propio Foucault ha insinuado reiteradamente alguno de ellos y multitud de monografías han subrayado los paralelismos (tanto los discutibles como los indudables) de Foucault con otros autores que toman el poder como objeto de sus investigaciones ${ }^{9}$. Lo que sí nos proponemos —en primer lugar- es subrayar la especificidad de la óptica foucaultiana aludiendo a alguna de las formas más habituales de tratar la temática del poder ${ }^{10}$.

Se puede afirmar que tal temática ha estado presente — de una u otra forma - a lo largo de la historia del pensamiento y ya desde sus inicios. Del poder tratan los sofistas, del poder habla Sócrates con Alcibiades en el diálogo platónico homónimo, al poder se refiere ocasionalmente la tragedia ática; San Agustín lo tematiza como una de las formas de libido, Santo Tomás ya introduce cuestiones de legitimidad y ejercicio, Maquiavelo indica cómo conseguirlo y cómo mantenerlo, Hobbes y Rousseau estudian el origen y postulan la forma óptima del poder, Montesquieu propone su legítima distribución, etc. Y en la ciencia social - a pesar del lamento de T. Parsons- ${ }^{11}$ el concepto de Poder ha estado

$9 \mathrm{Al}$ respecto se ha producido en los últimos años una gran cantidad de bibliografía, que ya tiende a ser redundante. En lo que toca a la temática aquí planteada, los estudios comparativos de la óptica de Foucault con las de Nietzsche, Marx y Weber constituyen, con mucho, la mayor parte. Citamos brevemente los que consideramos más importantes: MAHON, M.: Foucault's Nietzschean Genealogy, State University of New York Press, 1992; PizeK, J.: The use and Abuse of «Ursprung» on Foucault's Reading of Nietzsche, Nietzsche Studien 19/1990, pp. 462 ss.; ANSELL-PEARSON, K.: The Siqnifiance of Michel Foucault's Reading of Nietzsche: Power, the Subject, and Political Theory, Nietzsche Studien 20/1991, pp. 267 ss.; SMART, B.: Foucault, Marxism and Critique, Routledge and Keagan Paul, London 1983; BReuer, S.: Die Gesellschaft des Verschwindens, Junius Verlag, Hamburg 1993, pp. 41-64; BREUER, S.: «Sozialdisziplinierung. Probleme und Problemverlagerungen eines konzepts bei Max Weber, Gerhard Oestreich und Michel Foucault» en: SACHSSE/Tennstedt: Soziale Sicherheit und soziale Disziplinierung. Suhrkamp Verlag, Frankfurt/M 1986, pp. 45 ss.; NeuEnHaus, P.: Max Weber und Michel Foucault, Centaurus Verlag, Pfaffenweiler 1993; GoRdon, C.: «The Soul of the Citizen: Max Weber and Michel Foucault on Rationality and Government» en: WhimSTER/LASH: Max Weber, Rationality and Modernity, Allen Unwin, London 1987, pp. 293-316; O’NeILl, J.: «The disciplinary Society: from Weber to Foucault,» The British Journal of Sociology 37, n. ${ }^{\circ}$ 1, 1986, pp. 37-60; VAN KRIEKEN, R.: «The organisation of the Soul: Elias and Foucault on Discipline and the Self», Arch. europ. sociol., 31 (1990), pp. 353-371.

${ }^{10}$ La presentación es necesaria, aunque necesariamente rápida y parcial. Para valorar adecuadamente el trabajo de M. Foucault en torno al poder conviene referir — siquiera a base de breves trazos- el ámbito en el que se inserta y con el que rompe.

11 «El concepto de poder, lamentablemente, carece de arraigo en las ciencias sociales, tanto si se trata de sociología como de política». PARSONS, T.: «The distribution of power in American Society», World Politics, n. ${ }^{\circ} 10,1957$, p. 139. 
siempre presente y - a pesar de las diferentes definiciones- ha gozado de un rango especial.

Puede, en cualquier caso, aceptarse la apreciación de S. Lukes ${ }^{12}$ al respecto de que el concepto de poder tiene siempre carácter evaluativo y es una noción esencialmente contestable: es decir, que el concepto de poder surge siempre de una perspectiva moral y política particular y opera dentro de ella (carácter evaluativo), y que es uno de esos conceptos que «inevitablemente implican disputas interminables sobre sus usos correctos por parte de los usuarios» pues su aplicación es inherentemente discutible. Esta doble característica — harto incómoda, ciertamente- del concepto de poder explica la constante proliferación de definiciones, la inmensa producción de respuestas a tales definiciones, y el tono que alcanzan las disputas cuando de poder se trata.

A pesar de la señalada dificultad y aún conociendo de antemano la vulnerabilidad de la propuesta, nos atrevemos a sugerir —en base al uso que posteriormente nos ha de reportar- una tímida clasificación de las definiciones sociológico-políticas de poder en dos grandes tipos:

1. En el primer grupo hallamos definiciones de corte psico-social que destacan o sitúan en primer plano el conflicto de intereses y/o voluntades, para acabar definiendo el poder como la resolución exitosa del conflicto (es obviamente necesario el recurso a expresiones como imposición, manipulación, influencia) y aludiendo a un sujeto (individual o colectivo) que tiene tal poder de resolver el conflicto a su favor mientras otro sujeto (asimismo individual o colectivo) carece absoluta o relativamente de él.

De las definiciones que formarían parte de este primer grupo señalamos dos por su importancia:

En primer lugar la de Max Weber: «Poder (Macht) significa la probabilidad de imponer la propia voluntad dentro de una relación social, aun contra toda resistencia y cualquiera que sea el fundamento de esa probabilidad ${ }^{13} \gg$.

Como es sabido, Weber completa la definición de Poder (Macht) con las definiciones relacionadas de dominación (Herrschaft) y disciplina ${ }^{14}$ : «Por dominación debe entenderse la proba-

12 Lukes, S.: Power. A radical View, McMillan Press, London, 1974, p. 26. El texto, magnífico en su concisión y claridad, resulta imprescindible como trasfondo de lo que aquí enunciamos.

13 Weber, M.: «Economía y Sociedad». Fondo de Cultura Económica, México, 1984, p. 43.

14 Al respecto de los problemas que genera el uso del término Poder en los distintos idiomas, véase el esclarecedor artículo de ARon, R.: Macht, Power, Puissance: prose démocratique oи роésie démoniaque? Archiv. Europ. Sociol., V (1964), pp. 27-51. 
bilidad de encontrar obediencia a un mandato de determinado contenido entre personas dadas; por disciplina debe entenderse la probabilidad de encontrar obediencia para un mandato por parte de un conjunto de personas que, en virtud de actitudes arraigadas, sea pronta, simple y automática» ${ }^{15}$. Weber afirma que el concepto de poder (Macht) es sociológicamente amorfo, mientras el de dominación (Herrschaft) aparece precisado por la apelación al mandato y a la obediencia. Coincido con la apreciación de Weber al respecto del carácter amorfo de la noción de Poder. Es importante constatarlo pues tal apreciación afecta a todos los esquemas teóricos que definen el concepto de poder - dentro de este primer grupo- como resolución exitosa de un conflicto, latente o patente, de intereses o voluntades. De tal carácter amorfo da cuenta la consecución del argumento que con la definición de poder se inicia: de ella se deriva que alguien (el que impone la propia voluntad) tiene poder. Ahora bien, cabe preguntar ¿Qué tiene el que tiene poder? y entonces la respuesta nos sacaría del ámbito de la pregunta pues necesariamente tendría que aludir a algún otro elemento (prestigio, carisma, fuerza, dinero, fama...) que aparecería como previo al poder y como su condición de posibilidad.

Tal carácter amorfo del poder afecta a toda teoría o definición según la cual el poder se tiene. Entre ellas cabe señalar la teoría de las élites de C. Wright Mills o la teoría pluralista esquemáticamente representada por la definición de R. Dahl: «A tiene poder sobre B en la medida en que puede conseguir que B haga algo que de otra manera no haría» ${ }^{16}$ que ha generado en los Estados Unidos toda una tradición concernida por el estudio de «adopción de decisiones».

No continuamos añadiendo definiciones (que serían moderadamente redundantes). Señalamos tan sólo algo que, desde nuestra perspectiva de estudio, es fundamental: las teorías que inciden en el poder como resolución exitosa de un conflicto de voluntades $o$ de intereses:

a) Pretenden haber dado una definición general, universal e intemporal del poder: filosóficamente hablando diremos que tales definiciones presumen de haber hallado la substancia del poder, perseverante, por lo tanto, bajo todas las transformaciones históricas.

15 Weber, M.: Op. cit., p. 43.

16 Dahl, R.: «The concept of Power», en Bell/Edwards/Wagner: Political Power. A Reader in Theory and Research, MacMillan, London, 1969, p. 80. De C. WRIGHT MiLls, cabe citar: The power Elite, Oxford University Press 1956, esp. cap. 12; Power, Politics, People, Oxford University Press, 1963. 
b) Retienen tan sólo los aspectos negativos del poder: coerción, influencia, autoridad, manipulación, represión, etc.

2. El segundo tipo de definiciones del poder (siempre en el terreno sociológico-político) sería el de aquellas que no destacan el conflicto sino el consenso, que no aluden a intereses (y voluntades) enfrentados sino al «interés común» y a la «voluntad general» como soporte, fundamento y legitimación del poder. Se trata de una perspectiva que - en la época moderna - fue sugerida por Comte y que, con matices diferenciales, se percibe en T. Parsons, H. Arendt, J. Habermas, etc. ${ }^{17}$

La definición de T. Parsons «Poder es una capacidad generalizada de garantizar el cumplimiento de obligaciones vinculantes por parte de unidades dentro de un sistema de organización colectiva, cuando las obligaciones se legitiman mediante la referencia a su repercusión en los objetivos colectivos y donde en caso de actos recalcitrantes (recalcitrance) se dé presunción de ejecución a través de sanciones negativas sea quien sea el agente efectivo ( $a c$ tual) de tal ejecución» ${ }^{18}$ subraya el carácter colectivo de la toma de decisiones, el carácter general de la voluntad y el interés, el carácter sistémico del conjunto. «La conceptualización del poder de Parsons - afirma S. Lukes- asocia a éste con la autoridad, el consenso y la persecución de metas colectivas, y lo disocia de los conflictos de intereses y, en particular, de la coerción y la fuerza» ${ }^{19}$.

El poder se define desde la autoridad institucionalizada, como garante y portavoz de los intereses colectivos, de la voluntad general y, por lo tanto, del mantenimiento del sistema. Concebido como «a circulating medium, analogous to money» ${ }^{20}$, como medio generalizado a través del que se movilizan adhesiones y obligaciones en vista del interés de todos, el poder — como tal- carece de elementos negativos: la coerción, el uso de la fuerza, etc., quedan fuera de la definición de poder.

Algo similar sucede con la definición de poder de H. Arendt, tal y como se refleja en On Violence (y como hacen notar J. Habermas y S. Lukes) ${ }^{21}$ : «El poder corresponde a la capacidad hu-

$17 \mathrm{Y}$ esto, a pesar de que el último (J. Habermas) haya expresado sus críticas a la concepción de Parsons y Arendt y en él el consenso tenga carácter regulativo y no constitutivo.

18 Parsons, T.: «On the Concept of Political Power», en Sociological Theory and Modern Society, MacMillan, London, 1967, p. 308.

19 LuKes, S.: Op. cit., p. 28.

20 PARsons, T.: Op. cit., p. 306.

21 De H. AREndT: On Violence, Penguin, London, 1970; The Human Condition, University of Chicago Press, 1973. La crítica de Habermas en: Perfiles filosófico-políticos, Taurus, Madrid, 1984, pp. 200-222; la de Lukes, en Op. cit., p. 29 s. 
mana no sólo de actuar, sino de actuar de manera concertada. El poder no es nunca la propiedad de un individuo; pertenece a un grupo y existe tan sólo mientras el grupo permanece unido. Cuando decimos que alguien «está en el poder» nos referimos efectivamente a que tiene poderes de un cierto número de personas para que actúe en su nombre. En el momento en que desaparece el grupo que dio origen al poder en un principio (Potestas in populo: sin un pueblo o un grupo no hay poder), «su poder» se desvanece igualmente» ${ }^{22}$. Un poco antes señala: «Lo que presta poder a las instituciones y a las leyes de un país es el apoyo del pueblo, que a su vez es la continuación de aquel consenso originario que dio vida a las instituciones y a las leyes ${ }^{23}$.

En la definición de H. Arendt no hay mención a actitudes de dominio, coerción y violencia. Por esencia, según H. Arendt (por efecto de la definición, según nosotros) la violencia desaparece del ámbito del poder. Poder y violencia se repelen, pueden alternar pero no darse conjuntamente, hasta el punto de que «hablar de un poder no-violento es - según $\mathrm{H}$. Arendt- una redundancia». «Poder y violencia - continúa la autora - son contrarios, pues donde el uno domina de manera absoluta, la otra está ausente. La violencia aparece donde el poder corre peligro, pero abandonada a su suerte acaba con el poder» 24 .

La definición de H. Arendt no postula la primacía del sistema, como la anterior de Parson, pero coincide con él en el supuesto consensual. Para estos autores el poder es una fuerza exenta de rasgos negativos. Es el garante de la realización de la metas colectivas y actúa, por lo tanto, en beneficio de todos.

Cabe sospechar que, además de otros graves problemas como el de determinar lo que sean la voluntad colectiva y el interés general, la representación o el «mantenimiento del consenso», nos hallamos ante una solución terminológica de un problema nada conceptual. Es la definición la que aparta del poder cualquier rasgo negativo al establecer — sin más fundamento que una determinada opción sustentada en una determinada «perspectiva moral»la equivalencia poder $=$ consenso. El conjunto de categorías que acompañan a la noción de conflicto (violencia, lucha, fuerza, imposición...) es relegado a un exterior salvaje que, con respecto al poder, aparece como su estricto contrario; en cualquier caso como su negación o amenaza.

\footnotetext{
22 AREndt, H.: On Violence, ed. cit., p. 44.

23 Ibid, p. 42.

${ }^{24}$ Ibid., p. 56.
} 
Señalo a continuación una serie de rasgos que me merecen centrales en la concepción del Poder de H. Arendt pero que pueden hacerse extensivos (con inevitables cambios terminológicos) a todos los modelos teóricos que hacen del consenso el fundamento del poder, tanto de su origen o erección como de su mantenimiento o legitimidad. La presentación de tales rasgos - eminentemente crítica - no pretende tanto describir completa y correctamente el modelo como apuntar una serie de dudas, localizar puntos que, si no han de ser rotundamente negados, deben, cuando menos, pensarse en profundidad:

- Nos hallamos ante una definición del poder meramente política. Queremos decir con ello que H. Arendt sólo considera el poder en cuanto se localiza en (se asemeja a, participa de) el Estado. El hecho de que la equivalencia poder $=$ Estado sea la que ha prevalecido en la reflexión política moderna no indica un camino a seguir sino que sugiere una óptica deficiente o restringida. Pues no todos los poderes se localizan en el Estado: el Estado no es la síntesis del poder, no es el garante superior y el lejano fundamento de los «poderes» que se ejercen capilarmente, los que - por otra parteconstituyen el entorno inmediato de cada individuo y conforman el esqueleto de cada grupo.

- Todas las teorías que hacen del consenso el elemento constitutivo del poder nos sitúan, de nuevo, ante lo que podemos denominar la «invariante política»: la esencia del poder es consenso. El concepto así construido pretende universalidad por encima de las variaciones y accidentes históricos.

-El encuadre de la definición de poder de H. Arendt es valorativo y los contenidos de la misma son decididamente normativos. No sé - y tampoco es mi cometido decidirlo aquí- si localiza eficazmente el «debe ser» del poder. Lo cierto es que cabe dudar, como afirma Habermas, de la «utilidad científica del concepto» así construido $^{25}$. Más concretamente, elevado a concepto universal y definido en función del consenso, el concepto de poder carece de capacidad descriptiva frente a la multiplicidad de los poderes histórica y localmente constituidos.

- No es convincente la operación de soslayar, por obra y gracia de la definición, los aspectos negativos, el «lado oscuro» del poder. Y si es cierto que toda de-finición implica una previa de-cisión, es decir, un corte y un establecimiento de límites, también es cierto que la definición ha de ser comprensiva y no caprichosamente discri-

25 Habermas, J.: Op. cit., p. 208. 
minante. En cualquier caso, la dificultad de un trayecto no se elimina borrando en el mapa las dificultades orográficas. Y tal es lo que sucede con la definición de H. Arendt del poder: el trabajo cartográfico (la definición, el concepto) genera la ficción de una geografía amable; el territorio del poder es, sin embargo, espinoso, a menudo violento, eventualmente coercitivo. Forzando retóricamente el argumento, cabe afirmar que las ficciones de Kafka ${ }^{26}$ tienen mayor capacidad descriptiva y heurística que las definiciones consensuales del poder. Las primeras condensan fragmentos de experiencia vivida y construyen con ellos una hipérbole que se sabe tal: su técnica consiste en «llevar al extremo», en buscar «las últimas consecuencias», en buscar — de algún modo- el antitipo ideal. Las definiciones consensuales —en las antípodas de las ficciones kafkianas - proyectan una ficción inconsciente de apariencia conceptual cuya vinculación con la realidad (el poder tal y como cotidianamente se ejerce en condiciones socio-históricas concretas y entre actores históricos concretos) es más bien dudosa.

Los dos grupos mencionados completan —en lo esencial- el panorama de ofertas conceptuales al respecto del poder. He tomado como tales tan sólo aquellas que pretenden, cuando menos, estudiar el poder como elemento autónomo, si bien relativo al resto de elementos que se entrelazan a la estructura social. La omisión de las diversas corrientes de pensamiento marxista no se debe a olvido o a error. Comparten todas ellas la convicción inicial de que el poder depende - en mayor o menor gradode la infraestructura económica, es decir, que el poder y con él la totalidad del «nivel político» está determinado (o sobredeterminado) ${ }^{27}$. La concepción marxista del Estado y del poder político nos llevaría pues a enfrentarnos al concepto de dominación implicado en la lucha de clases, al propio concepto de clase, y al marco económico en el que las clases se definen.

De lo dicho hasta ahora podemos resumir en breves trazos unas características generales que han de servirnos como trasfondo crítico:

a) El concepto de poder es, a pesar de Parsons, un concepto con prestigio evidente $-\mathrm{y}$ creciente- en las ciencias sociales.

${ }^{26}$ Una lectura socio-política de las ficciones de Kafka en: GonZÁLEZ GARCíA, J.M.: La máquina burocrática. Afinidades electivas entre Max Weber y Kafka. Ed. Visor, Madrid, 1989; cfr. mi crítica a este texto en la Revista Internacional de Filosofía Política, n. ${ }^{\circ} 3$, primavera 1994.

${ }^{27}$ Especialmente interesantes en este sentido: Poulantzas, N.: Poder político y clases sociales en el estado capitalista. Ed. Siglo XXI, Madrid, 1975, pp. 117-146; Miliband, R.: El estado en la sociedad capitalista, Ed. Siglo XXI, México, 1985. 
b) La investigación se centra principalmente en torno al concepto de poder político.

c) La imagen o la figura del Estado preside la práctica totalidad de las reflexiones sociológicas al respecto del poder.

d) Del poder se pretende un concepto unívoco que garantice la universalidad del objeto.

Y si es cierto que estos rasgos generales componen las coordenadas básicas entre las que se ha mantenido la disputa teórica al respecto del poder a lo largo de los últimos decenios, no puede sino concluirse que la reflexión de Michel Foucault ha debido resultar sorprendente, difícilmente aceptable en el marco teórico prefijado, resueltamente disidente para con las categorías usuales y, por lo tanto, original, caprichosa e incluso extravagante.

Del poderoso y complejo entramado crítico, histórico y descriptivo que completa el trabajo foucaultiano con respecto al poder, extraemos una idea general que ha de servir de falsilla hermenéutica: Foucault no afronta el estudio del poder con vistas a una teoría política, no se trata tanto de una teoría del poder destinada a convertirse en alternativa a las ya existentes sino una analítica del poder orientada al estudio de las técnicas de constitución del sujeto en su relación con «los otros». De este modo, el ámbito del poder se inserta en el proyecto general de M. Foucault en la forma de una «Ontología histórica de nosotros mismos en las relaciones de poder que nos constituyen como sujetos actuando sobre los demás ${ }^{28}$.

Para entender esta perspectiva e insertarla en la obra foucaultiana con todas sus consecuencias - y son muchas- es preciso repudiar alguno de los esquemas que una lectura superficial genera, alguno de los prejuicios que una lectura parcial sustenta. Fundamentalmente dos: el de un Foucault concernido únicamente por el rigor de la institución, por la proximidad del aparato (sea éste manicomio, cárcel o sanatorio), por el ambiguo prestigio del edificio y su entorno humano y epistémico; y el de un Foucault subyugado por la omnipresencia de un fantasma (el Poder) al que habría dotado de dimensiones cósmicas negándole además las características que habrían permitido someterlo conceptualmente: un poder que se hallaría «überall und nirgendwo» ubicuo y esquivo, inmediato y distante, trascendente e inmanente. Muchas de las críticas a la filosofía de Foucault (sin duda la de Habermas, y tras él las de Dews, Breuer, Honneth, etc. $)^{29}$ ponen en juego los dos prejuicios antes mencionados

${ }_{28}$ Foucault, M.: en Dreyfus/Rabinow, Op. cit., p. 237; Morey, M.: en Tecnologías del yo, Paidós, Barcelona, 1990, p. 25.

${ }_{29}$ Habermas, J.: Der philosophische Diskurs der Moderne, Suhrkamp Verlag, Frankfurt/M 1985, pp. 279-343; Breuer, S.: Op. cit.; Dews, P.: Op. cit.; Honneth, A.: Kritik der Macht, Suhrkamp Verlag, Frankfurt/M, 1986. 
para apoyar sobre ellos (y agotar en ellos) el fecundo trabajo foucaultiano sobre las técnicas de subjetivación en el ámbito del poder.

Tan sólo un ejemplo: Habermas imputa a Foucault la inmoderada extensión al conjunto social - como «moderna tecnología de dominación»- del «arquetipo de la institución cerrada que Foucault descubre primero en el mundo del asilo reconvertido en clínica» y que «retorna en las figuras de la fábrica, de la prisión, del cuartel, de la escuela y del colegio militar» ${ }^{30}$ constituyéndose en signo de victoria de una razón reglamentadora. En el mismo texto le acusa de haber heredado de Nietzsche un concepto «absolutamente asociológico de poder» que, de forma insidiosa, cobra esa imagen camaleónica y difusa a la que antes hacíamos referencia. Escuchemos a Habermas: «lo único que perdura es el poder, el cual en el cambio de procesos de avasallamiento anónimos aparece bajo máscaras siempre nuevas» ${ }^{31}$. Tal poder, substantificado y deificado (o demonizado) es, a juicio de Habermas, el «irritante concepto básico» (irritierende Grundbegriff) de la crítica de Foucault a la razón.

¿Cómo combinar esa imagen proteica del poder tal y como Habermas lo percibe en la obra de Foucault con la sospecha, propiamente foucaultiana, de que el Poder «no existe»? ¿Cómo compatibilizar ese fantasma del poder ubicuo y eterno, mil veces transformado por la multiplicación de las máscaras, con la precisa localización y la discreta presencia que Foucault adscribe a las «prácticas de poder»?

Lo que sucede es decididamente simple en cuanto a su explicación, y evoca una pretérita disputa — acendrada y nada estéril— acaecida en Europa en torno al siglo XIV: Habermas, fascinado por el anejo prestigio del concepto, no puede entender que la palabra rehúse captar la sustancia universal del poder (que ésta resida en la «imposición de la propia voluntad» o en el «consenso» sería posteriormente discutible); Foucault, indiscretamente nominalista, niega a la vez la substancia, la universalidad y el concepto: «Hay que ser nominalista, sin duda: el poder no es una institución, y no es una estructura, no es cierta potencia de la que algunos estarían dotados: es el nombre que se presta a una situación estratégica compleja en una sociedad dada ${ }^{32}$.

La presunta «universalidad» del poder, su fatal omnipresencia no responde al modelo de una substancia que se transforma y a la vez se mantiene, idéntica a sí misma, bajo las apariencias diversas. No, en cada sociedad, en cada época, hay una forma particular de poder, local y lo-

30 Habermas, J.: Op. cit., p. 287.

31 Ibid., pp. 297 s.

32 Foucault, M.: Histoire de la sexualité. La volonte de savoir, Gallimard, París, 1976 (Trad. esp. Historia de la sexualidad I. La voluntad de saber, Siglo XXI, Méjico 1977, en adelante HSI), p. 113. 
calmente analizable; lo cual quiere decir que en toda sociedad hay relaciones de poder, no que cada sociedad esté sometida a la forma universal del poder y estructurada por ella. En palabras de Foucault: «La palabra "poder" amenaza con introducir varios malentendidos. Malentendidos acerca de su identidad, su forma, su unidad. Por poder no quiero decir "el Poder", como conjunto de instituciones y aparatos que garantizan la sujeción de los ciudadanos en un Estado determinado. Tampoco indico un modo de sujeción que, por oposición a la violencia, tendría la forma de la regla. Finalmente, no entiendo por poder un sistema general de dominación ejercida por un elemento o un grupo sobre otro, y cuyos efectos, merced a sucesivas derivaciones, atravesaría la totalidad del cuerpo social. El análisis en términos de poder no debe postular, como datos iniciales, la soberanía del Estado, la forma de la ley o la unidad global de una dominación; éstas son más bien formas terminales» ${ }^{33}$.

Así pues, el poder aparece en toda (y en cada) sociedad porque sociedad es un conjunto de relaciones de poder: «Vivir en sociedad es vivir de tal manera que sea posible la acción de unos sobre la acción de los otros. Una sociedad sin relaciones de poder no puede ser sino una abstracción» ${ }^{34}$. Relaciones de poder, por lo tanto, y no poder (ni Poder). Foucault afirma que el poder no existe ni con mayúscula ni con minúscula (something called Power, with or without a capital letter, wich is assumed to exist universally in a concentrated or diffused form, does not exist ${ }^{35}$. Del poder sólo existen relaciones concretas y actuales; más el ejercicio que la lejana posibilidad. De ahí que Foucault afirme, de forma ciertamente sorprendente que él no habla de poder: "Yo apenas utilizo la palabra poder, y en las ocasiones en que lo hago se trata simplemente de resumir y abreviar la expresión que constantemente uso: relaciones de poder» ${ }^{36}$. Relaciones de poder, por lo tanto, y no poder sin más: multiplicidad de relaciones y no una substancia esquiva y ubicua; omnipresencia de las relaciones (pues una sociedad no es sino un conjunto de relaciones) y no omnipresencia de un objeto persistente y enigmático. «El poder está en todas partes —afirma Foucault-, no es que lo englobe todo, sino que viene de todas partes» ${ }^{37}$.

A partir de estas notas, breves pero suficientemente clarificadoras, resulta más fácil acotar el terreno en el que vamos a situarnos. Recurriendo a los siempre gratos y esquemáticos pares de oposición diremos que se

33 Ibid., p. 112.

34 En Dreyfus/Rabinow: Op. cit., pp. 222 s.

35 Ibid., p. 219.

36 BECKER, H.: Freiheit und Selbstsorae. Gesprach mit Michel Foucault, Materialis Verlag, Frankfurt, 1985, p. 19.

${ }^{37}$ HsI., p. 113. 
trata - en el caso de Foucault- no de una metafísica sino de una física del poder; no de una substancia (en respuesta a la pregunta ¿qué es?) sino de múltiples relaciones (que responden a la pregunta ¿cómo se ejerce?); no de un presupuesto universal sino de infinidad de resultados locales.

Este triple desplazamiento sitúa las coordenadas fundamentales de la reflexión foucaultiana sobre el poder en un marco ajeno al de las anteriormente descritas. Es, por lo tanto, difícil afirmar tanto el parentesco como la oposición de la filosofía de Foucault para con las de Weber, Arendt, Parsons, Wright Mills, Habermas, etc. En la medida en que no comparten el mismo territorio, las coincidencias terminológicas (el uso por parte de todos ellos de la palabra «poder») no pueden sino conducir a graves confusiones. Si para algunos autores «Poder» aspira a rango conceptual, para Foucault es apenas una palabra, es más una abreviatura que no apunta permanentemente hacia el mismo objeto. Por ello, aunque resulte paradójico, la única respuesta cabal a la pregunta ¿qué es el poder? en la perspectiva foucaultiana, sería la siguiente: el poder no es.

Ahora bien, si la palabra «poder» designa tan sólo el mapa estratégico de las relaciones en una sociedad y en un momento dados, entonces no carece de sentido el gesto por el cual Foucault rompe la periodización durante mucho tiempo habitual de su obra, e incorpora los ensayos sobre la locura y la enfermedad a la nómina de estudios sobre el poder, insistiendo no tanto en la común pertenencia a la fase «genealógica» como en el hecho de que - de una u otra forma - estos escritos afrontan el problema de las relaciones entre los hombres en el seno del conjunto social, es decir, el tema-problema del poder. De esta forma, el tema del poder se convertiría en el primero (tal vez en el principal) del conjunto de la obra de Foucault. Tal conclusión, que puede no ser del todo errónea, resulta manifiestamente incompleta si nos fiamos de las reiteradas apreciaciones de Foucault al respecto de la continuidad y coherencia de su proyecto general y extraemos de ellas lo que afecta a la temática del poder. En todas las últimas intervenciones (artículos, entrevistas...) en las que Foucault se refiere tanto a sus libros pretéritos como a su trabajo en curso (La Historia de la sexualidad) aporta una serie de elementos que ubican la temática del poder - entendido como relaciones- en un horizonte teórico más complejo. También más interesante. Cito alguna de esas últimas intervenciones ${ }^{38}$ :

1. «En la segunda parte de mi trabajo he estudiado la objetivación del sujeto en lo que yo denominaría «prácticas escindentes». El

38 A pesar de la, tal vez inmoderada, longitud del conjunto de citas, creo que es interesante apreciar tanto lo común a las diversas relecturas que Foucault propone de su propia trayectoria intelectual como los matices diferenciales que se perciben en algunos testimonios. 
sujeto es dividido, sea al interior de sí mismo, sea escindido de los otros. Este proceso hace de él un objeto. La división entre el loco y el cuerdo, el enfermo y el hombre de buena salud, el criminal y el «buen chico» ilustra esta tendencia» ${ }^{39}$.

2. «Sigue (en el orden de su trabajo) una ontología histórica de nosotros mismos en nuestras relaciones a un campo de poder, en el cual nos constituimos en sujetos actuando sobre los otros $»^{40}$.

3. «Michel Foucault ha intentado también analizar la constitución del sujeto tal y como éste aparece al otro lado de una división normativa y así deviene objeto de conocimiento a título de loco, de enfermo o de delincuente y esto a través de prácticas como las de la psiquiatría, la medicina clínica o la penalidad» ${ }^{41}$.

4. «Siempre ha consistido mi problema en determinar las relaciones entre el sujeto y la verdad: ¿cómo entra el sujeto en un juego concreto de verdad? (...) Esto me ha llevado a plantear el problema saber/poder, que no es para mí el problema fundamental sino un instrumento que posibilita estudiar las relaciones entre el sujeto y los juegos de verdad de una forma que me parece adecuada (...) Lo que he querido mostrar es cómo el sujeto se constituye de determinadas formas - que encierran juegos de verdad y prácticas de poder- como loco o sano, como delincuente o no-delincuente ${ }^{42}$.

5. «Lo que me ha interesado (...) han sido precisamente las formas de racionalidad aplicadas por el sujeto humano sobre sí mismo (...). ¿Cómo se constituye el sujeto a sí mismo como objeto de conocimiento posible? ¿A través de qué formas de racionalidad y de qué condiciones históricas? ¿Y, finalmente, a qué precio? (...) En el estudio de las relaciones de poder no he intentado construir una teoría del poder. He pretendido estudiar cómo ha sido guiada la reflexión del sujeto y el discurso de la verdad: ¿cómo puede decir el sujeto la verdad sobre sí mismo? (...) En el límite diría que el poder, como cuestión autónoma, no me interesa ${ }^{43}$.

6. «Lo que he estudiado han sido tres problemas tradicionales: 1) ¿cuáles son las relaciones que tenemos con la verdad a través del conocimiento científico, con esos «juegos de verdad» que son tan importantes en la civilización y en los cuales somos, a la vez, su-

39 En Dreyfus/Rabinow: Op. cit., p. 208.

40 Ibid., p. 237.

41 En: Huisman, D (Dir.): Dictionnaire des Philosophes, P.U.F., París 1984, p. 942.

42 En BeCKER, H.: Op. cit., pp. 17-18.

43 Foucault, M.: Structuralism and Post-Structuralism (Interview with G. Raulet) Telos, Vol. 16, n. ${ }^{\circ} 55$ Spring, 1983, pp. 202-203. 
jeto y objeto?; 2) ¿cuáles son las relaciones que entablamos con los demás a través de esas extrañas estrategias y relaciones de poder?; 3) ¿cuáles son las relaciones entre verdad, poder e individuo?» ${ }^{44}$.

Creo que sabrá disculparse la longitud de las citas. El interés del conjunto teórico que diseñan las hace necesarias no sólo para reclamar una nada dudosa coherencia de la obra de Foucault sino para imprimir un cambio de perspectiva que disipe algunas filiaciones sospechosas, disperse algunos lugares comunes y haga vanas muchas críticas.

Lo que permite esta secuencia de citas en las que Foucault propone unas claves de relectura de su obra (pretérita y «presente») es interpretar esta última en base, no a una cronología simplista, ni a una sucesión metodológica cada vez más dudosa, sino, mucho más importante, en base a una serie de presupuestos ideológicos, ideas rectoras o convicciones fundamentales, algunas de las cuales no han merecido por parte del autor un tratamiento esmerado, aunque sí suficientes alusiones como para que no resulte excesivamente arriesgado proponerlas aquí como horizonte de interpretación.

En primer lugar, un inveterado nominalismo (la expresión no entraña ningún matiz crítico; pretende solamente alcance descriptivo). Las expresiones hatualmente utilizadas por Foucault inducen decididamente a error si no se tiene en cuenta el supuesto nominalista. Foucault trabaja sobre el «saber», el «poder», el «sujeto», la «sexualidad». Es cierto si (y sólo si) se acepta que tales expresiones no tienen valor conceptual, que no designan universales. El saber sólo existe en cuanto discurso: «lo ya dicho y ya escrito», localizado por lo tanto, ubicado en un determinado lugar y momento. Lo mismo cabe decir del poder: no se trata de ese fantasma que insidiosamente se distiende y se apodera de la totalidad de la historia. Habermas afirma al respecto de Foucualt: «Lo único que perdura es el poder». Se trata de un defecto de intelección, de un error de perspectiva. El poder ni perdura ni puede perdurar porque «el poder» no existe. No se trata de un abuso retórico. Es preciso asumir la expresión literalmente. Independientemente de lo que propongan las teorías políticas al uso, para Foucault -y para el que esto suscribe- no existe «el poder»: existen relaciones de poder, prácticas de gobierno, acciones localizadas y a menudo minúsculas que no conforman una unidad de la que cabría extraer concepto o narrar su historia. No. La historización del poder en los escritos de Foucault hay que entenderla como la producción de una historia de las prácticas de gobierno: prácticas que se suman, que se

44 Foucault, M.: Tegnologías del yo. Ed. cit., p. 150. 
yuxtaponen, que se sustituyen, que se intersectan; todo menos recogerse en ese lugar común, hospitalario y sintético, que sería el poder. Nominalismo, por lo tanto, y en sentido estricto; no hay un poder que vincula la pluralidad de los ejercicios de gobierno; no hay una sexualidad que recorre la multiplicidad de cuerpos; no hay un saber que inspira la masa de los discursos.

En segundo lugar, y en íntima relación con lo anteriormente dicho, cabe señalar en Foucault una vocación decididamente pluralista ${ }^{45}$. Y en este sentido, podremos hablar de verdades y prácticas de gobierno, de sujetos y ejercicios de poder, de sexualidades y discursos. El nominalismo no conduce, como pudiera pensarse, al escepticismo (sólo para una concepción universalista cabría establecer tal ecuación) sino a la observación minuciosa y pertinaz de las múltiples actividades — sean de índole discursiva o práctica - que se amalgaman en la historia. Para el nominalista que es Foucault, la historia no produce síntesis sino dispersión. Por ello hay que desconfiar de las unidades apresuradas; por ello la pregunta reiterada de Foucault: ¿a qué actividades —múltiples, dispersas, autónomas- aludimos cuando decimos poder?, ¿a qué procesos cuando hablamos de sujeto?, ¿a qué discursos cuando decimos saber?

En tercer lugar, y en un sentido preciso, un arraigado historicismo: que todo se produce en la historia es la negación estricta de que la historia configura el todo. La historia no es, para Foucault, ni continuista, ni progresiva, ni dialéctica. Estas tres actitudes teóricas suponen una pauta o hilo conductor, en el extremo una actividad teleológica. Diremos - por seguir negando unidades apresuradas - que, para Foucault, no existe la historia. Expresiones como «producir historias» o «escribir ficciones», con las que Foucault caracteriza su propio trabajo acentúan la inexistencia de una historia con orden y sentido. Incluso la Historia tiene su historia $^{46}$. Y lo que nunca produce la historia es algo así como la forma universal del poder, o de la sexualidad, o del sujeto. Acontecimientos ínfimos, sistemas complejos de gestión y gobierno, prácticas para usar el cuerpo en busca de placer o descendencia, discursos explicativos o códigos de conducta: esto es lo que se produce en la historia. La actividad de vincular, establecer divisiones de época o estilo, la impostación de dirección y/o sentido es siempre actividad de interpretación, actividad del historiador o del filósofo.

45 No confundir el término, utilizado aquí en su acepción convencional, con el uso que de él hace la autodenominada Teoría pluralista del poder (R. Dahl et alii).

46 A este respecto es fundamental el texto de Foucault Nietzsche: la genealogía, la historia (en MP, pp. 7-29) que desvela además las nada arcanas relaciones entre el pensamiento del propio Foucault y el de Nietzsche. 
Creo que estas tres actitudes (nominalismo, pluralismo, historicismo) reflejan conjuntamente el trasfondo del trabajo foucaultiano sin necesidad de recurrir reiterativamente a conceptos sacralizados (genealogía, arqueología) o a expresiones decididamente polisémicas o equívocas (irracionalismo, relativismo, escepticismo, positivismo ${ }^{47}$.

$\mathrm{Al}$ asumir el contenido de estas tres actitudes fundamentales — presentes y perceptibles a lo largo de la trayectoria foucaultiana- se puede detectar claramente el equívoco que subyace a todos los comentarios (entusiastas o adversos) que toman por objeto la teoría, la crítica o la historia del poder en la obra de Foucault y una vez constatado tal equívoco, se debe intentar leer el trabajo foucaultiano desde otra óptica, cuyo punto de partida viene sugerido por el mismo autor en los textos que más arriba hemos reproducido: el intento de producir una historia de los modos de subjetivación (necesariamente en plural) del ser humano en nuestra cultura. Una vez negado el carácter transcendental del sujeto se tratará de ver cómo se constituyen tipos de subjetividad en ámbitos complejos de relaciones: juegos de verdad y prácticas de poder.

La aparente diversidad de la obra de Foucault se desvanece. Cobra por el contrario una apariencia monótona. Desaparecen del horizonte los grandes temas, los suntuosos proyectos, y permanece tan sólo una perseverante investigación de las relaciones que, a lo largo de la historia y de forma distinta, han construido sujetos al interrogar por determinadas verdades, al gobernar determinados hábitos, al problematizar determinadas actitudes, al establecer determinados límites o al regular determinadas convivencias.

La mirada del genealogista — sea Nietzsche o Foucault - entrenada en la recepción del detalle, no se conforma con síntesis apresuradas: bajo la aparente evidencia de la locura descubre los hilos de mil historias; bajo la severidad del poder, el eco de mil batallas. Y en el centro de esas historias y batallas se encuentra el individuo interrogado y gobernado, sujeto-objeto de verdades y poderes, constituido una y otra vez - de formas siempre diferentes - en marcos diversos de pensamiento y acción.

Si ésta es la rejilla adecuada para leer el trabajo de Foucault —él mismo lo confirma-, entonces podrá apreciarse la distancia del filósofo francés para con todos los ensayos que pretenden reducir la pluralidad de las relaciones de saber-poder a la disciplina de una «forma general», ya sea la del consenso (Arendt, Parsons) ya sea la de la resolución exitosa del conflicto (Weber, Dahl, Mills). Se comprende también su esceptismo frente a todas las majestuosas figuras que —en el seno de una teoría-

47 Véase Veyne, P.: «Foucault révolutionne l'histoire», en: Comment on écrit l'histoire, Seuil, París, 1978, pp. 203-242 (hay traducción castellana). 
aparecen como el centro desde el que el poder irradia, se expande y pretende perpetuarse: figuras entre las que el Estado tiene un lugar preeminente. Y asimismo su distancia de una - posible o imposible- Teoría Política que intentase captar el poder en sí mismo.

Frente a esas tres direcciones que - desde hace por lo menos un siglo- ensayan las diferentes Teorías, Filosofías y Sociologías políticas, Foucault opone otro tipo de trabajo. Quede bien consignado: no se opone a ellas, no compite con ellas, no se enfrenta a ellas; apenas las considera si no es requerido a ello en contadas entrevistas. Y sus dudas al respecto de las posibilidades de un estudio centrado en esas grandes figuras y conceptos (Estado, Poder, Consenso, Violencia) no se expresan sino en la forma oblicua e implícita de un trabajo paralelo.

Con la previsión del error al que puede inducir el uso del singular en la palabra sujeto, creo que, para resaltar el carácter original y específico del rastro foucaultiano, habría que comenzar todo texto sobre el problema del poder en Foucault anteponiendo sus propias palabras: «No es el poder sino el sujeto el tema general de mis investigaciones».

Como hemos anticipado, se habla de sujetos cuando de poderes se habla; aún mejor, de individuos insertos en prácticas de gobierno, de vigilancia, de disciplina, de castigo; de individuos que ocupan su lugar en determinadas instituciones y están sometidos a determinados códigos; de individuos a los que «su trabajo hace libres» y otros a los que «su verdad hace cautivos...».

La pregunta por el poder permite especificar ese ámbito de relaciones en el que se constituye el sujeto al actuar -individual o colectivamente- unos individuos sobre otros. En este sentido cabe afirmar — con Foucault- que la pregunta por el poder descubre un instrumento para analizar la constitución del sujeto.

Y, entretanto, ¿qué ha sucedido con el Poder?: «El poder es algo que no existe. Esto es lo que quiero decir: la idea de que hay en un sitio determinado, o emanando de un punto determinado, algo que sea un poder, me parece que reposa sobre un análisis trucado, y que, en todo caso, no da cuenta de un número considerable de fenómenos. El poder, en realidad, son unas relaciones, un conjunto más o menos coordinado de relaciones $»^{48}$.

48 Foucault, M.: Saber y Verdad, Ed. de la Piqueta, Madrid, 1985, p. 132. 